\title{
A new code SORD for simulation of polarized light scattering in the Earth atmosphere
}

\author{
Sergey Korkin*a,b, Alexei Lyapustin ${ }^{\mathrm{b}}$, Aliaksandr Sinyuk ${ }^{\mathrm{c}, \mathrm{b}}$, and Brent Holben ${ }^{\mathrm{b}}$ \\ ${ }^{a}$ USRA GESTAR, 7178 Columbia Gateway Drive, Columbia, MD, USA 21046; ${ }^{\mathrm{b}}$ NASA GSFC, \\ 8800 Greenbelt Rd., Greenbelt, MD, USA 20771; '`Sigma Space Corp., 4600 Forbes Blvd., Lanham- \\ Seabrook, MD USA 20706
}

\begin{abstract}
We report a new publicly available radiative transfer (RT) code for numerical simulation of polarized light scattering in plane-parallel atmosphere of the Earth. Using 44 benchmark tests, we prove high accuracy of the new RT code, SORD (Successive ORDers of scattering ${ }^{1,2}$ ). We describe capabilities of SORD and show run time for each test on two different machines. At present, SORD is supposed to work as part of the Aerosol Robotic NETwork ${ }^{3}$ (AERONET) inversion algorithm. For natural integration with the AERONET software, SORD is coded in Fortran 90/95. The code is available by email request from the corresponding (first) author or from ftp://climate1.gsfc.nasa.gov/skorkin/SORD/.
\end{abstract}

Keywords: polarized radiative transfer, successive orders of scattering, open-source scientific software

\section{INTRODUCTION}

Successive orders of scattering is a method of numerical simulation of light scattering in atmosphere-surface system. The core of the method is iterative computation of the next scattering order from the current one. Iterations start from single scattering approximation, which has a simple analytical form.

Polarization of light is included in the method using the Stokes vector, $[I, Q, U, V]$, and the Mueller matrix formalism. Under some circumstances, neglecting of the effect of polarization causes significant errors in numerical simulation of radiative transfer ${ }^{4,5}$. The effect of polarization must be accounted to accurately process data even for non-polarimetric systems ${ }^{6}$. From polarization, one gets information not available from analysis of the total intensity only ${ }^{7}$. Several polarimeters were developed for atmospheric science ${ }^{8,9}$. All these systems require fast and accurate simulation of polarized light scattering in atmosphere.

The known monochromatic vector radiative transfer equation ${ }^{10}$ (RTE) provides theoretical basis for the method of successive orders. Boundary conditions imposed on the RTE define irradiance on top of atmosphere (TOA) and reflectance from the bottom (BOA). The RTE has one derivative over dimensionless optical thickness, and two integrals, one over cosine of the zenith angle, and one over azimuth. Fourier expansion of the RTE solution allows for analytical integration over azimuth. For the zenith integral, Gauss quadrature is used. Integration over optical depth is performed numerically as well. Thus, there are four important input parameters to define accuracy and run time for the method. They are the number of Fourier moments, $M$, for integration over azimuth; the order of Gauss quadrature, $N$, for the zenith integration; the number of layers, $L$, for vertical integration over optical depth; and the number of scattering orders, $S$, taken into account.

At present, the method of successive orders is well developed. Lenoble ${ }^{2}$ (see page 47) summarized advantages and weaknesses of the method. For the reader's convenience, we copy them here (in italic), followed by comments (in regular font) arose from our own experience. The main advantages are:

Better physical understanding of the problem than in most methods, because the photon is followed at each scattering process. Specifically, it means that one can estimate contribution of each azimuthal (Fourier) component and each scattering order in simulation. Given the fact, that high scattering orders possess smooth angular distribution, they do not have azimuthal components of high Fourier order. Vice versa, one needs high Fourier moments only for low scattering orders. Using automatic convergence criterion, one can automatically prevent computation of high orders and Fourier harmonics with insignificant contribution. This saves time and harmless for accuracy. We discuss this in the next section. 
Possibility to handle inhomogeneous or homogeneous atmospheres by the same program. More importantly, run time does not depend on number of optical layers, i.e. layers with different optical parameters (single scattering albedo, scattering law), because of numerical integration over optical depth.

The intensity is obtained for as many depths and directions as points chosen in the quadrature formula. Noteworthy, the solution can be obtained at arbitrary view direction inside atmosphere using integration of the source function ${ }^{11,12}$ or brutal interpolation ${ }^{13}$;

In a homogeneous semi-infinite atmosphere, when intensity has been computed for single scattering equal one, it may be obtained immediately for all other values of single scattering albedo using Eq.(3.50) from Lenoble ${ }^{2}$. We have never benefited from that in our particular study.

In our research, we learned a few more advantages. For instance, the method is relatively simple from the coding point of view. It does not require external mathematical libraries. The developer has full control over optimization and debugging. It also makes the code more portable. The code in scalar (neglecting polarization) and vector (with polarization) forms looks very similar. If it is created in one form, it is not hard to create another one (downgrade from vector to scalar is of course easier). Having both scalar and vector codes might be useful in retrieval algorithm because it is sufficient to start minimization with a faster scalar code. The vector form is to be used at final stage for accuracy.

The following shortcomings exist:

The convergence of series of successive scattering becomes very slow when single scattering tend to one (very weak absorption) and if, at the same time, optical depth is large the computation time becomes exorbitant; but it may be improved by various methods of acceleration of the convergence or by use of asymptotic expressions for the higher order scattering terms; in the latter case it has been applied to semi-infinite atmospheres. In general, this is correct. However, most of the considered benchmarks have very weak or no absorption. Some benchmarks are defined for optical depth 5 and even 10. We do not feel the method suffers from low accuracy. Indeed approximation of high scattering orders by geometric series is helpful (we discuss this further in the text).

The program must be run completely for each value of $\mu_{0}$ (cosine of solar zenith angle). This is correct and becomes important when one generates look-up tables for many solar zeniths. However, running the whole RT code for each solar angle may become an advantage. The number of Fourier terms depends on solar zenith angle. For the case of the Sun overhead, the solution is azimuthally symmetric and require only one Fourier moment, $m=0$. The Fourier series gets longer on the Sunset. Thus running the RT code for several solar angles at a time with the same number of Fourier moments may turn out redundant for high Sun and/or insufficient for low Sun. On the contrary, automatic convergence traces accuracy for each solar angle separately.

We add two more disadvantages to the list above. First, in the framework of the method, it is not possible to split surface from atmosphere. This becomes important when only atmosphere parameters are retrieved, while the surface is assumed known. The situation is typical for ground based sun photometers and satellite remote sensing over ocean. In part, one can solve the problem by precomputing and storing surface in memory. Second, because of numerical integration over the whole atmosphere, it does not seem possible to compute different optical layers in atmosphere independently. This is desirable for cirrus cloud cases, when RT in cirrus itself is simulated using high order of Gauss quadrature (hundreds of ordinates), while molecular atmosphere above and below the cirrus cloud is computed using low order (ten).

At present, there are several well-developed RT codes based on the method of successive orders. Min and Duan have developed a vector RT code, SOSVRT ${ }^{12}$. They proposed a technique to speed up computations for thick media ${ }^{14}$. Zhai and co-authors using their successive orders of scattering code ${ }^{15}$ have developed a coupled atmosphere-ocean model ${ }^{16}$. They applied their model to ocean color remote sensing problem ${ }^{17}$. They also developed a correction technique for the second scattering order ${ }^{18}$. The code described in Lenoble et al. ${ }^{13}$ was used to solve variety of RT problems (see references in their paper).

Nevertheless, there are pitfalls. The main of which is lack of analysis of accuracy of the successive orders of scattering method in different scenarios. Naturally, the above-mentioned authors give examples of accuracy in their papers. However, not a single code of this kind participated in recent thorough RT codes intercomparison ${ }^{19}$. We believe this our paper is the first one that reports results of the RT codes intercomparison for all the variety of published scenarios, in particular for scattering by dust and clouds. Our team has participated in the mentioned intercomparsion ${ }^{19}$ with our own RT code IPOL (Intensity and POLarization) ${ }^{20}$. IPOL uses the method of discrete ordinates and proved high accuracy in all scenarios defined in the intercomparison. 
In 2010, the RT code SOSVRT participated in intercomparison organized by Kokhanovsky ${ }^{21}$ and was found accurate. Nevertheless, this benchmark is limited to homogeneous atmospheres over a black surface. The intercomparison defined only three cases: a simple case of Rayleigh scattering with no depolarization factor; and two complicated cases of light scattering by large particles (coarse fraction of spherical aerosol and cloud of water droplets). These cases are important and complicated for simulation of the total intensity, but the degree of polarization is small. Light scattering by spheroids was not considered as well.

Another problem is, as usual, availability and support. SOSVRT is available by request from the authors ${ }^{22}$. However, we are not sure about the level of support and documentation of the code.

Because the following reasons, we decided to create our own RT code. We selected the method of successive orders of scattering because of the mentioned advantages, as well as based on the experience of the AERONET team with RT code of this kind ${ }^{13}$. The experience is overall positive, but distant support by the developer located in France is not always convenient. Using local code is obviously preferable.

A natural negative side of a young software is short history of use. In order to get critical comments from all interested users, we deliberately make our RT code SORD publicly available and intent to provide as much support as we only can. Nothing reveals problems as efficient as using the software by someone other than the developer. However, there is an essential step in testing of any new software, which we can take on our own. This step is scrupulous validation against reliable, i.e. published, benchmarks. To this we devote our article.

\section{FEATURES AND VALIDATION OF THE CODE}

\subsection{Features of the code}

The RT code SORD incorporates several features essential for atmospheric and terrestrial applications. They include atmosphere with arbitrary number of optical layers; polarized light scattering by spherical particles or spheroids; molecular (Rayleigh) scattering with arbitrary depolarization factor; arbitrary solar and observation geometry without interpolation between Gauss ordinates; surface reflection from land and waved ocean surface. The code has several widely used models for surface reflection: black, isotropic (Lambertian), waved polarized ocean with ${ }^{23}$ and without ${ }^{24}$ specified wind azimuth, scalar ${ }^{25,26}$ and vector ${ }^{27}$ land models. Linear mixture of land and ocean simulates observation at coastal sites for AERONET. A convenient interface allows adding new surface models. Single scattering albedo smaller than one simulates gas absorption.

For numerical integration over optical thickness, the method of successive orders computes radiation at many levels in atmosphere. In our code, we follow Lenoble et al. ${ }^{13}$ (see p.497, Eq.(66)). Namely, we spread levels over optical depth (microlayers) equidistantly. At each level and direction, we know the value of superposition (integral over zenith angle) of previous scattering order and the phase matrix. For integration over each microlayer, we take an average of the two neighboring superposition integrals outside the integral over optical depth, and integrate the exponential (Bouguer) decay factor analytically. Further, the result is accumulated over microlayers as usual. The solution is available at all levels. However, for ground and satellite remote sensing, SORD provides only reflected (at TOA) and transmitted (at $\mathrm{BOA}$ ) radiation. The code also computes ascending and descending fluxes at TOA and BOA.

The fourth component of the Stokes is small in atmosphere ${ }^{19}$ and often neglected ${ }^{4}{ }^{13}$. It does not give any significant effect on the intensity and linear polarization, but allows for noticeably faster computations and smaller memory requirements. For linear polarization, the frame of reference must be clearly defined. The frame of reference defines the sign of $Q$ and $U$. If two RT codes differ in sign, it does not yet mean that one of the codes is wrong. The sign is important when a new vector surface is built in, because the code and surface frames of reference must match. If one fails to do that, the error for the total intensity likely will not be crucial, unlike for degree polarization. In addition, the sign of $Q$ and $U$ is important in polarized retrieval algorithms that use information from $Q$ and $U$. Difference in sign may "pull" the retrieval algorithm in a wrong direction.

In our code, we strictly adhere definition from Hovenier et al. ${ }^{28}$ We refer the reader to that book for details. Here we briefly mention a few important features (with reference to page and, optionally, equation number in Hovenier et al. ${ }^{28}$ ):

- The angle $\chi$ in $\tan 2 \chi=Q / U$ (page 6, Eq.(1.25)) is measured in the anti-clockwise direction, as viewed in the direction of propagation of the light beam (page 4, after Figure 1.2); 
- The angle of rotation is positive in the anti-clockwise direction when looking in the direction of propagation (page 11). The rotation matrix is defined as in Eq.(1.51);

- On output, the code gives $Q$ and $U$ components in the meridian plane, i.e. the plane through the direction of observation and the local normal (axis) $Z$ (page 66);

- The $Z$ axis points up from the ground towards the space (page 67, Figure 3.2);

- The zenith angle is counted from the positive $Z$ direction. Zenith angle $0^{\circ}$ corresponds to ascending radiation (detected by satellites), while $180^{\circ}$ corresponds to radiation descending on the ground (page 66);

- Relative azimuth is measured clockwise when looking in the positive $Z$ direction ("up", page 66).

Light scattering by large particles is challenging. In order to speed-up computations, modern RT codes truncate the scattering function and apply single (or sometimes double) scattering correction ${ }^{29}$ as post processing. Our experience ${ }^{30}$ showed that such an approach gives huge errors in the aureole area. Because we are planning to use the code for observations from ground, we refrained from using any truncation technique ${ }^{31}$ until further detailed research.

The method of successive orders benefits from using geometric progression to simulate infinite number of scattering orders $^{2}$. For high orders, the ratio of the current and previous scatterings tends to be constant ratio of geometric sequence. The constant weakly depends on two scattering orders, assumed high enough, but of course strongly depends on the view angle and optical depth. SORD computes scattering orders for each Fourier harmonic until some scattering order becomes small. At this point, we stop computation of orders. From the last two orders, we get the ratio of the geometric sequence. Using the ratio, we approximate all higher orders from the last computed to infinity as geometric sequence. At the zenith and nadir directions, we compute the ratio only for azimuthally average intensity, Fourier moment $m=0$. For all other Fourier moments, the intensity equals zero along the normal direction, both up and down. We skip them. For the degree of linear polarization, similar problem occurs at the neutral points. After some numerical tests, we avoided this problem by applying the same ratio for intensity, $I$, and polarization components, $Q$ and $U$.

It is important to define criterion on what order is high enough to replace the residual orders by the geometric sequence. For that, we have an input parameter for "weakness" of magnitude of $I$. We define this parameter by absolute value, not as relative value. Clearly, it is not necessary to meet relative criterion for an negligibly small value. Another advantage of this approach arises from the fact that high scattering orders are smooth functions over angle and do not contain high Fourier harmonics. The opposite is true as well: only low scattering orders require long Fourier series. Thus, the stop criterion expressed in absolute values traces both convergences of the Fourier series and orders of scattering. On input, the user may define $M=2 N-1$ (the highest possible Fourier order equals total number of ordinates per whole sphere; -1 accounts for the fact that Fourier orders are often start from $m=0$ ). Convergence will stop automatically at some sufficient Fourier order, maybe long before the input $M$ is reached (e.g., for the Sun at zenith only one Fourier harmonic is computed). On the negative side of this approach, the user must have some idea about magnitude of solution in his case of interest. The magnitude depends on all optical parameters of atmosphere and surface, geometry of observation and irradiation, and flux (value and units) on TOA. In each particular case, the user chose the stop criteria from trial-anderror.

As opposed to definition in absolute units, the relative stop criterion defined for each Fourier moment individually allows cutting high scattering orders, but not the redundantly high Fourier terms. Same definition but related to accumulated Fourier series allows cutting both weak scattering orders and Fourier harmonics, but prohibits parallel computation of the Fourier moments. A somewhat better idea would be to precompute the single scattering, SS, plus the azimuthally averaged, AA, high orders and relate any current Fourier term with $m>0$ and corresponding scattering order to the precomputed "SS+AA" value. We have not implemented this idea yet. At this point, the absolute stop criterion is our choice.

With the stop criterion thus defined, only three accuracy parameters remain. They are the number of ordinates, $N$, the number of layers, $L$, and the stop criterion itself. The sufficient number of the Fourier moments, $M$, and scattering orders, $S$, are selected automatically. Note that high scattering orders require lower order of Gauss quadrature because of smoothness over zenith angle. To the best of our knowledge, no RT code including SORD uses this feature for acceleration.

In the rest of the paper, we focus on comprehensive validation and performance analysis of the new RT code SORD. 


\subsection{Validation methodology}

Comparison against published benchmarks is the most reliable and essential step for validation of a new software. We present results of 44 tests, including 39 published benchmarks. In three cases, we have used the code RT $3^{32}$, and for the other two our own code IPOL ${ }^{20}$ carefully validated during the recent RT codes intercomparision ${ }^{19}$. The code comes with a testing subroutine that automatically reads all necessary data, runs and validates SORD against them. The result of comparison is printed out in log files. Using the log files, we plotted figures for the paper.

We analyze two relative errors. One is for the total intensity, I. Another is for the degree of linear polarization,

$$
P=\sqrt{Q^{2}+U^{2}} / I, \quad 0 \leq P \leq 1 .
$$

For the $I$, the relative error between the benchmark, $I_{B}$, and SORD, $I_{S}$, is defined by absolute value

$$
\delta I=100 \% \cdot\left|I_{B}-I_{S}\right| / I_{B} .
$$

For the $P$, we use the following definition

$$
\delta P=100 \% \cdot\left|P_{B}-P_{S}\right|
$$

where $P_{B}$ and $P_{S}$ are degree of linear polarization, Eq.(1), for benchmark and SORD, respectively. We use \% in Eq.(3) for consistency with Eq.(2). For example, for $P_{B}=0.13=13 \%$ and $P_{S}=0.12=12 \%$, one gets $\delta P=1 \%$. As follows from Eqs. (2) and (3), we skip the sign.

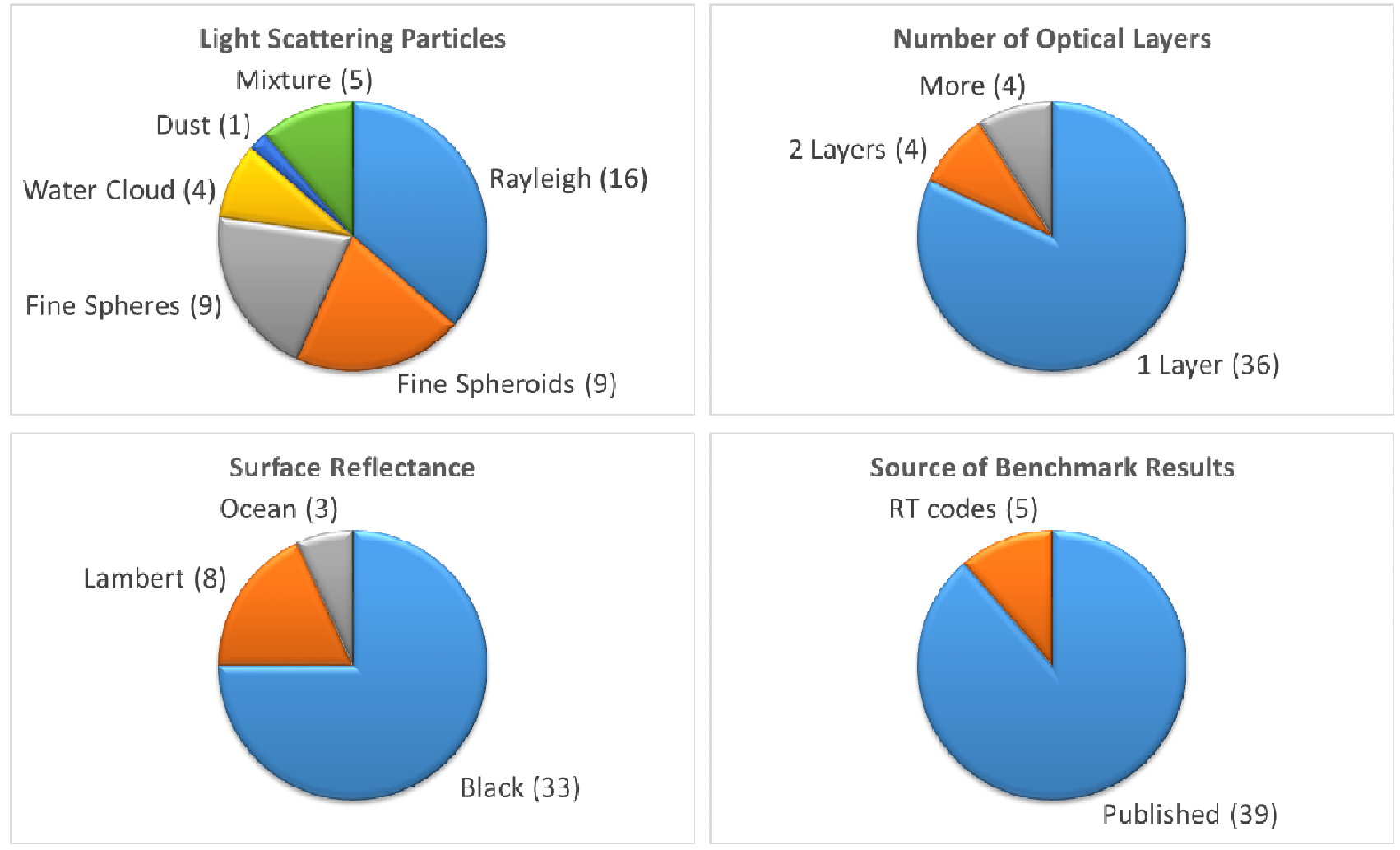

Figure 1. List of scenarios itemized by topic: scattering type, number of layers, surface, and source of benchmark results. The number in brackets, (X), shows how many scenarios has a particular feature. For example, the bottom right diagram shows that 39 of the benchmarks are published. There are 44 scenarios total.

In order to make the analysis meaningful, we must define some level of sufficient accuracy for $I$ and $P$. This is important not only to prove accuracy of the new code, but also because nobody wants to get excessively accurate result at the expense of computation time. For that, we refer the reader to Table 1 from Diner et al. ${ }^{9}$ (see p.8429). The table shows polarimetric uncertainty for several space borne instruments: Polarization and Directionality of Earth's Reflectances 
(POLDER) - 2\%; Multiangle SpectroPolarimetric Imager (MSPI) - 0.5\%; Aerosol Polarimeter Sensor (APS) - 0.2\%. Hasekamp and Langraf ${ }^{7}$ (see Conclusion, p.3343) set the same requirement $0.002=0.2 \%$ for the relative Stokes parameters, $q=Q / I$ and $u=U / I$, and no more than $1 \%$ for the intensity, $I$.

For reference, we recommend the reader to keep in mind $0.1 \%$ as error threshold for both $\delta I$, Eq.(2), and $\delta P$, Eq.(3).

\subsection{Benchmark scenarios}

This section lists all used benchmarks with references to literature. References to RT3 $3^{32}$ and $\mathrm{IPOL}^{20}$ means the authors generated unpublished benchmark results using the codes RT3 and IPOL, respectively. The complete list of tests is:

- $\quad$ Single layer pure Rayleigh atmosphere ${ }^{19,21,33}$;

- Single layer Rayleigh atmosphere with non-zero depolarization factor or SSA $<1^{19,32}$;

- Single layer Rayleigh atmosphere over isotropic surface ${ }^{19,32}$;

- $\quad$ Single layer Rayleigh atmosphere over waved ocean surface ${ }^{19,34}$;

- Multilayer Rayleigh atmosphere ${ }^{19,33}$;

- Single layer atmosphere with fine or mild (haze) spherical particles over black or isotropic surface ${ }^{19,35,36}$;

- Single layer atmosphere with randomly oriented spheroids over black or isotropic surface ${ }^{19,37,38,39,40}$,

- Atmosphere with two layers containing aerosol and Rayleigh mixture over isotropic surface ${ }^{20,35,41}$;

- Single layer atmosphere with coarse fraction of spherical aerosol ${ }^{21}$;

- Single layer atmosphere with coarse fraction of non-spherical aerosol (dust) ${ }^{19}$;

- Single layer atmosphere with cloud of liquid water droplets ${ }^{19,21}$.

Note that sign of $Q$ and $U$ components in SORD differs from the one used in RT3 and reported in Kokhanovsky ${ }^{21}$.

The pie-plots in Figure 1 show itemized list of scenarios. For instance, the "Light Scattering Particles" diagram shows that SORD was tested for different scattering laws: from simple Rayleigh scattering, to complicated cases of clouds and large dust particles. Rayleigh scenarios hold the majority because of simplicity of computations (see original ${ }^{42}$ and improved $^{43}$ tables for Rayleigh scattering) as well as because Rayleigh scattering is the most polarized one. As opposed to that, there are just a few reliable benchmark results for polarized light scattering by large particles available from literature ${ }^{19,21}$. Noteworthy, there is an obvious lack of published non-isotropic land benchmarks. In the nearest future, we plan to fill the gap using our new code SORD as well as IPOL.

In addition to Figure 1, we report the following range of the solar and view geometries, total optical depths, and surface reflection coefficients (for isotropic surface reflection only):

- Solar zenith angle: from $0^{\circ}$ to $\sim 85^{\circ}$;

- View zenith angle: from $0^{\circ}$ to $180^{\circ}$ step $1^{\circ}$, except for $90^{\circ}$ (horizon). There are a few points with view zenith angle $90^{\circ} \pm 0.3^{\circ}$ (Gauss nodes in tests against RT3);

- Relative azimuth (difference between view and solar azimuths): from $0^{\circ}$ to $360^{\circ}$, step $5^{\circ}$;

- Total atmosphere optical thickness: from 0.2 to 10 ;

- Reflection coefficient up to $0.7=70 \%$ reflection.

We conclude with certainty that the selected set of tests is representative. Agreement with the benchmark results within the accuracy $0.1 \%$, set above for intensity and liner polarization, guarantees accuracy of our new code SORD.

\subsection{Results of validation}

In the error analysis below, we frequently mention the term "point". Each point corresponds to a fixed solar and view geometry in a given scenario. For example, if some benchmark contains 1 solar angle, 3 upward view zenith angles, 3 downward zenith angles, and 5 azimuths, this gives us 45 different geometries or 45 points. Two different benchmarks 
with 45 geometries in each give 90 points. The total number of points involved in our 44 tests is 16888 . We are certain this statistics is sufficient to check accuracy of our RT code.

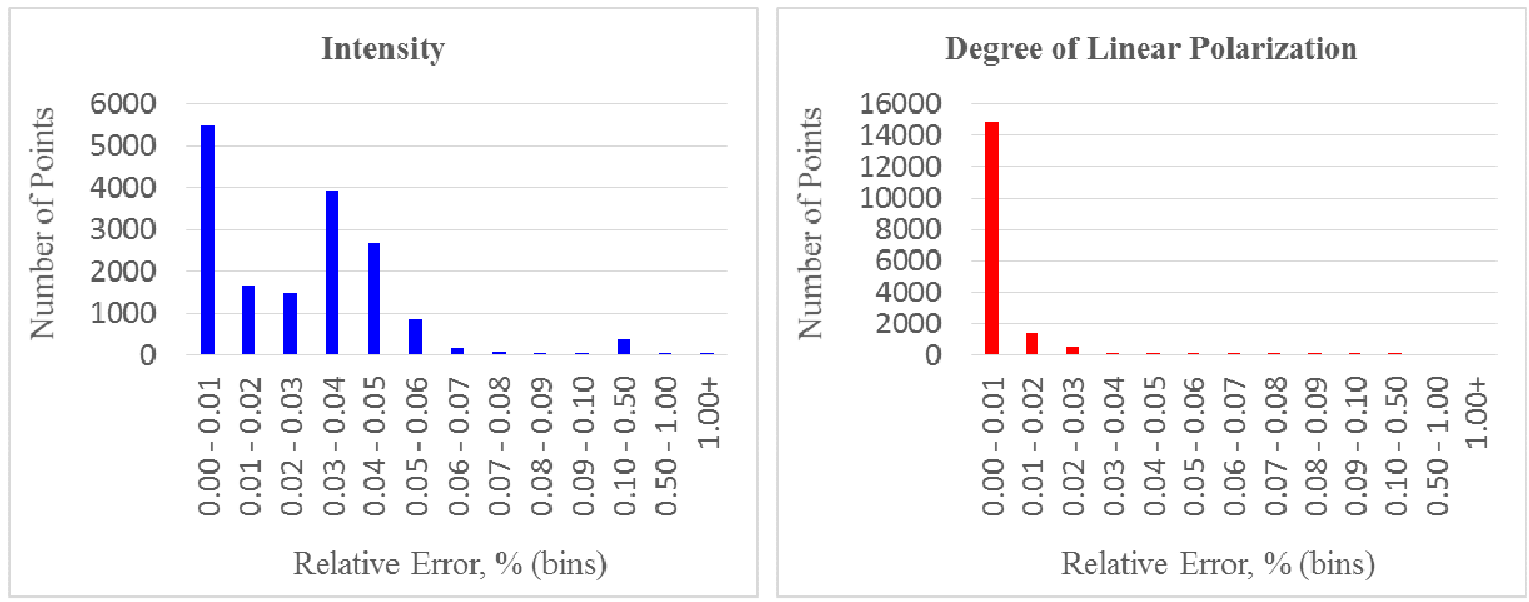

Figure 2. Distribution of number of points over bins of relative error, in $\%$, for the total intensity (left) and degree of linear polarization (right). The total number of points (different geometries for different scenarios) is 16888 .
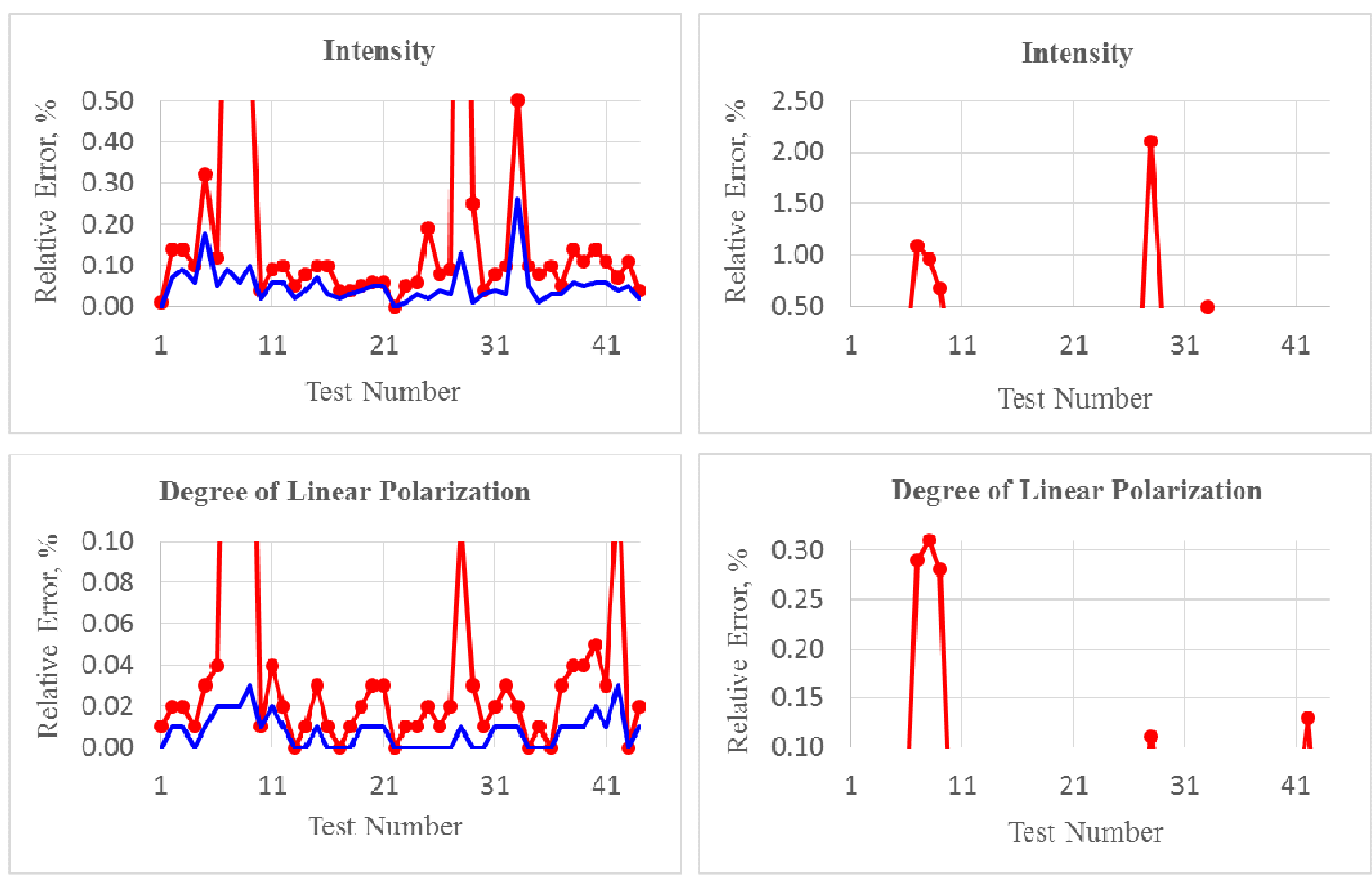

Figure 3. Maximum (red line with dot markers) and average (blue solid line) absolute values of relative errors for intensity (top row) and degree of linear polarization (bottom row) in each test. Right column depicts a few points with higher errors for intensity not shown in figures from the left column.

Figure 2 shows distribution of relative error, in \%, for the total intensity, $I$ (left), and the degree of linear polarization, $P$ (right). For instance, from the left chart one can see that almost 4000 points for $I$ show relative deviation in the range $0.03-0.04 \%$. The degree of linear polarization converges faster than the total intensity. The right histogram in Figure 2 shows the vast majority of relative errors for $P$ fall below $0.03 \%$, while for $I$ there is still considerable amount of points with errors up to $0.06 \%$ and even $0.5 \%$. We conclude that convergence of intensity to the desired precision guarantees convergence of degree of linear polarization at least to the same level of accuracy, Eq.(3). 
Figure 3 shows the maximum and average relative errors, in \%, for intensity (top left and top right) and degree of linear polarization (bottom left and bottom right) in each test. For better appearance, the right column shows peak errors for $I$ not shown in the left column. Outliers, i.e. points with high (compared to other data) errors, appear under the following circumstances:

- Direction of observation is very close to horizon: view zenith angle is $89.70^{\circ}$ and $90.30^{\circ}$. In this case, we observe $\sim 1 \%$ error in intensity. For polarization the error is still within the desired limit $0.1 \%$;

- A bump at 0.1-0.5\% in Figure 2 (left) is, in part, due to light scattering by dust. For better accuracy, this particular points may require more ordinates;

- A $2.1 \%$ difference in $I$, depicted on the top-right plot in Figure 3 corresponds to the coarse fraction of aerosol $^{21}$ in case of view zenith complimentary to solar zenith angle and relative azimuth $180^{\circ}$ (anti-solar observation of descending radiation). Note, for the direct solar beam, the error is $0.07 \%<<2.1 \%$. The light is almost unpolarized, so $\delta P$ is $0.0 \%$. For better accuracy, this single point requires more Fourier moments and hence more run time. Other points become "overconverged", i.e. excessively accurate. Hence, if this particular geometry of observation is important, it should be computed separately;

- Cases with total atmosphere optical depth $\sim 10$ also possess $\sim 0.5 \%$ difference (for descending radiation), contributing to the bump on the left chart in Figure 2. For reflectance, the same level of error is smaller, $\sim 0.10$ $0.15 \%$. For both ascending and descending radiation, the level of error for $\delta P$ is $0.0 \%$. This confirms efficiency of our implementation of the geometric sequence approach for optically thick atmospheres.

We conclude this section with the following general statistics. For the total intensity, $97.5 \%$ of points match the criterion of $0.1 \%$ relative error, while for the degree of liner polarization the number is $99.8 \%$ for the same threshold. For vast majority of points, the agreement is much better than $0.1 \%$. We emphasize that the error analyses shown here is based on a particular set of input parameters responsible for accuracy and run-time, listed above. By changing these input parameters, it is possible to match the user's "runtime vs accuracy" requirements even better. The overall statistics proves high accuracy of SORD in the conducted tests.

\subsection{Timing}

Papers rarely report run time for benchmarks ${ }^{19,21}$ because it depends on too many factors. Among them are developer skills (we deliberately put the "human factor" first), hardware and software used, targeted accuracy, selected numerical method of solution, compiler keys and many others. Despite these difficulties, we would like to report the run time, in seconds, for all 44 benchmarks.

In order to make the timing factor meaningful, we give parameters of the two machines used to test our code:

- Machine 1: Intel i7-2720QM CPU, 2.2GHz, Windows 764 bit; Intel Visual Fortran Compiler 11.0 .072 integrated with Microsoft Visual Studio 2008. In Visual Studio, we enabled the "Maximize Speed" option located under Configuration Properties\Fortran\Optimization. Further, we refer to this machine by the compiler name, "ifort".

- Machine 2: Intel Xeon E7-4890 v2 CPU, 2.8 GHz, Linux 2.664 bit; The Portland Group Fortran 90/95 compiler 7.1-4 with the following compiler keys: -03 -Mipa=fast, inline = Msmartalloc. We refer to this machine as "pgf 90".

The run time was measured for each test using the intrinsic Fortran function CPU_TIME on both machines. For each test, the reported run time is an average value over 10 runs. At a single run, all 44 tests were performed. Thus, for a particular test, runs were not consecutive. For instance, between two consecutive runs of the $5^{\text {th }}$ test, all remaining 43 tests were performed. This leaves almost no chance for a compiler to store arrays, defined for the $5^{\text {th }}$ test, in memory and thus accelerate performance beyond the user control.

As noted above, computation of each scattering order involves two numerical integrations. One over zenith angle, another one over optical depth. For any given direction of observation, scalar (dot) product integrates over the zenith angle. The code use dot product many tens of times for all involved directions of observation and at all levels inside atmosphere, including TOA and BOA. Because of that, dot product takes noticeable amount of time. One must tune it appropriately for maximum performance. 
There are different ways to organize dot product of two vectors, $\mathrm{A}$ and $\mathrm{B}$, in Fortran: a) to use intrinsic DOT_PRODUCT (A, B) function; $b$ ) too use intrinsic SUM $\left(A *_{B}\right)$ function combined with element-by-element multiplication; $c$ ) to use external function such as DDOT from $\mathrm{BLAS}^{44}$, or create your own. We compared $(a)$ versus $(b)$ on both machines and received the same run time in all the tests. Below we compare SUM (A*B) versus (c) DDOT.
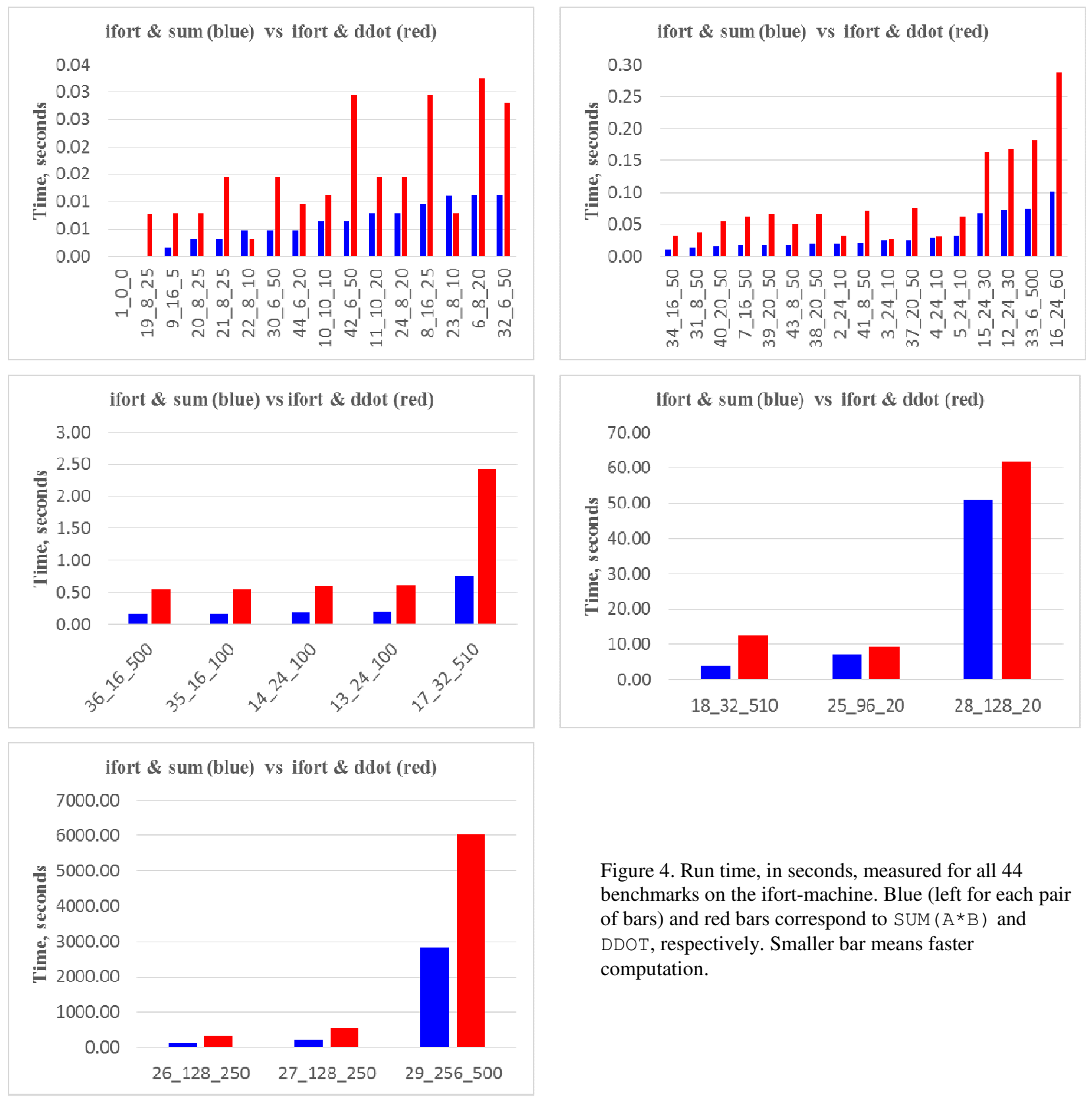

Figure 4. Run time, in seconds, measured for all 44 benchmarks on the ifort-machine. Blue (left for each pair of bars) and red bars correspond to $\operatorname{SUM}(A \star B)$ and DDOT, respectively. Smaller bar means faster computation.

Besides hardware and software, one must mention at least two more parameters. They are the number of layers for integration over optical depth, and the number of ordinates for integration over zenith angle. Computation burden is directly proportional to first power of the number of layers, and second power of the number of ordinates. We show these parameters on X-axis of Figures 4 and $\mathbf{5}$ in the form of NB_NH_NT. In this notation, NB is the number of benchmark test (for reference), $\mathbf{N H}$ is number of ordinates per hemisphere (2NH is the total number of ordinates), and $\mathbf{N T}$ is the number of layers used for numerical integration over optical thickness of atmosphere. For example, 29_256_500 means that test No.29 was computed with 256 ordinates per hemisphere (512 total), and 500 microlayers. This test is the 
slowest of all. On the contrary, the first test, $1 \_0 \_0$, validates single scattering in analytical form. It does not require numerical integration over zenith angle ( 0 ordinates) and over optical thickness ( 0 microlayers). Y-axis in Figures 4 (for the ifort-machine) and 5 (for the pgf90-machine) show run time in seconds.
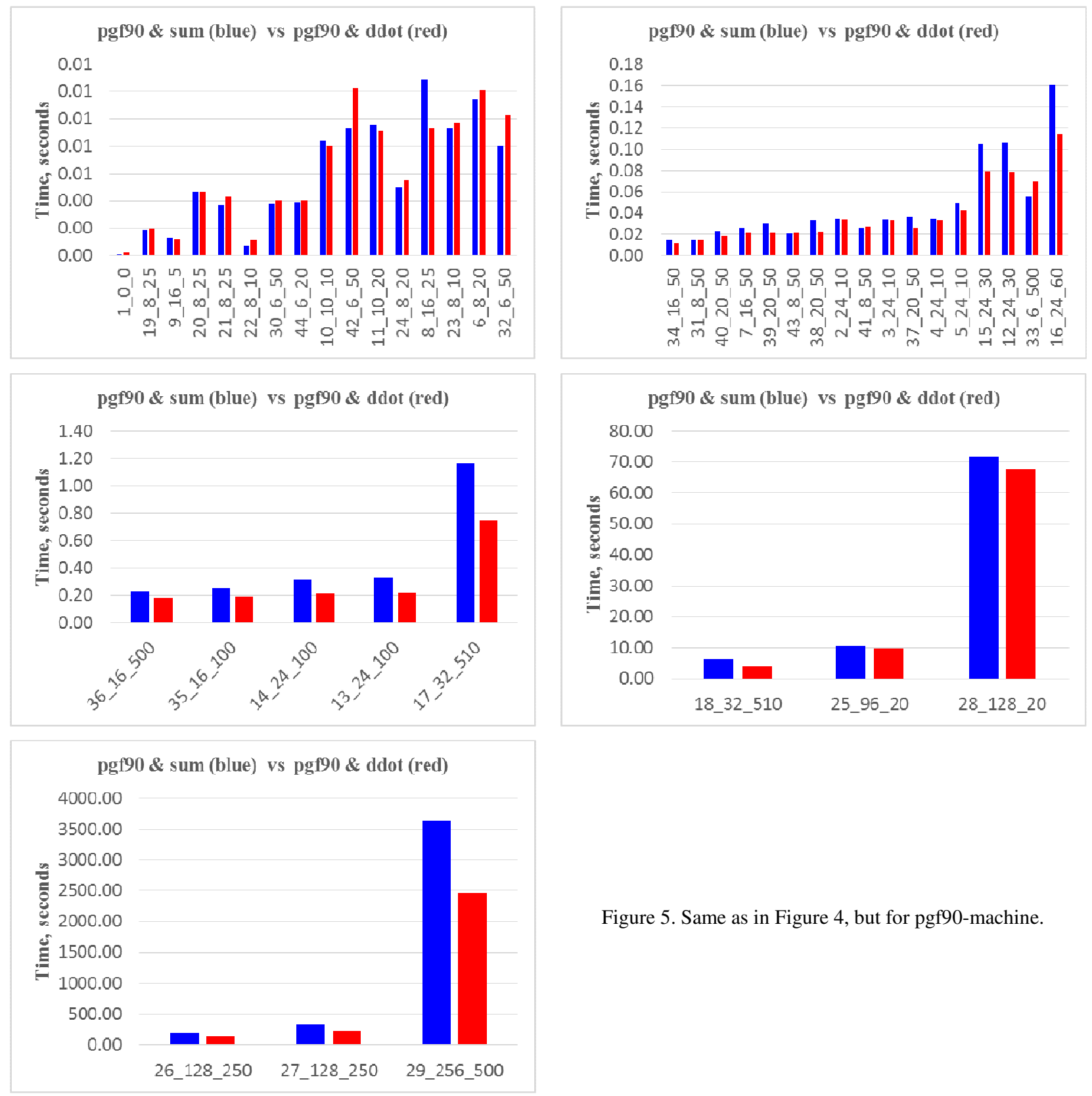

Figure 5. Same as in Figure 4, but for pgf90-machine.

For convenience, we grouped all the results in different subplots, 5 per machine, in such a way that all measured run times are legible. On both Figures 4 and 5, blue (left in each pair of bars) and red bars correspond to $S U M(A * B)$ and DDOT, respectively. We noticed that intrinsic function $\mathrm{SUM}(\mathrm{A} * \mathrm{~B})$ works noticeably faster on the ifort-machine compared to DDOT. However, for the pgf90-machine, the effect was the opposite. In many cases, DDOT (red bars) showed better performance (see Figure 5). This is explained by different algorithms used in SUM and DDOT. For SUM, the source is not available, as it is for DDOT. For better performance, DDOT uses the unrolled loops technique. For details, we refer the reader tom "Basic Loop Unrolling" chapter in Dowd ${ }^{45}$. DDOT uses unrolled loops with a factor $5^{44}$, which may or may not be efficient. For potential problems with this technique, we again refer the reader to the chapter 
"Unrolling by the Wrong Factor" from the same book ${ }^{45}$. It seems like Intel compiler on Intel CPU has some built in optimization, like selecting the best factor to unroll the loops. Hence using of intrinsic function is recommended for Intel compilers running on Intel machines. For other options, like non-Intel compiler on Intel machine, we recommend to make a trial-and-error research and determine the unroll factor which shows the best performance. For reference, we also compared SUM $\left(A{ }^{\star} B\right)$ on ifort versus DDOT on pgf90 and plotted the result in Figure 6. The fastest benchmarks are not shown.
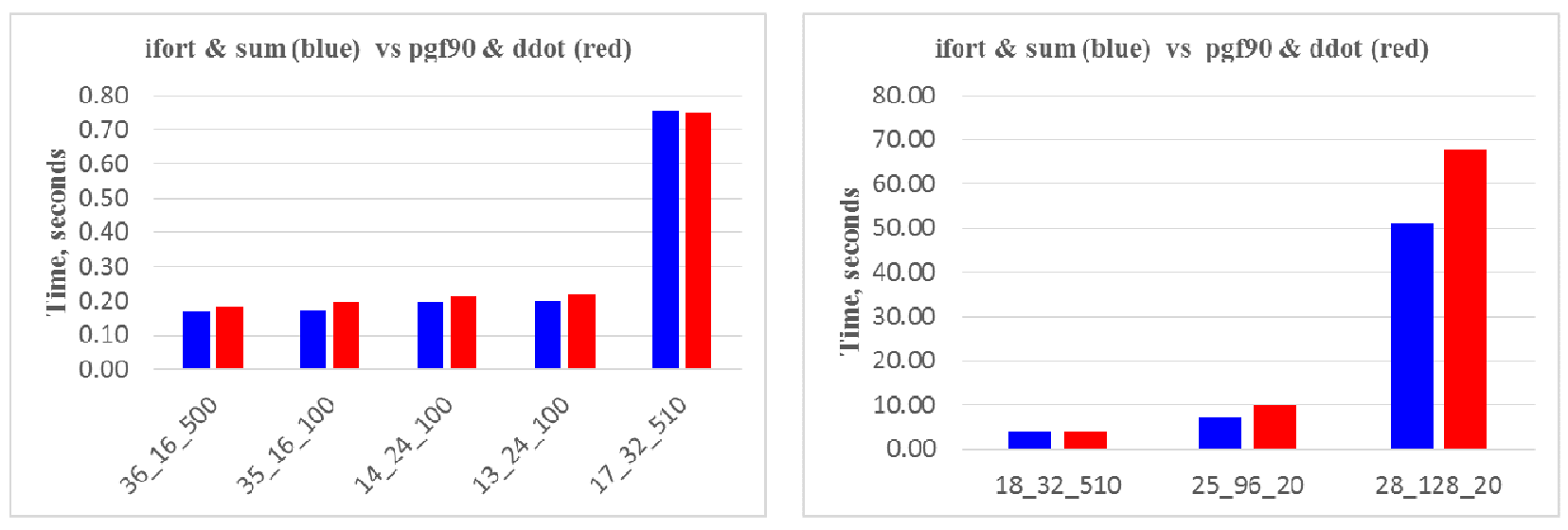

ifort \& sum (blue) vs pgf00 \& ddot (red)

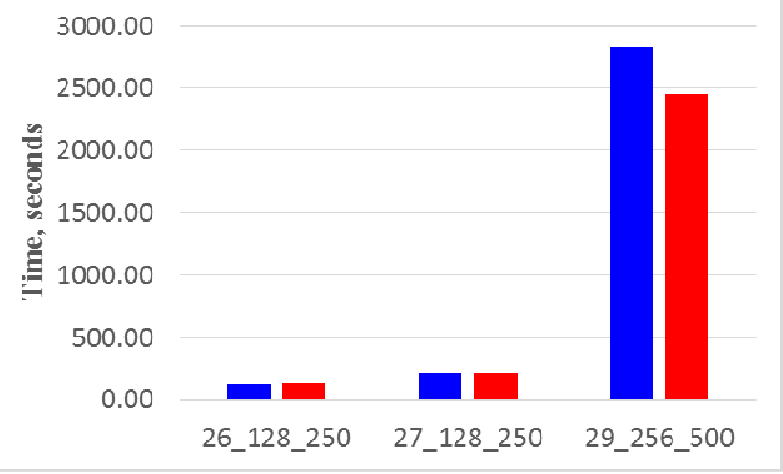

Figure 6. Run time for ifort \& sum vs pgf 90 \& ddot and 3 grades of tests: fastest (top left figure), moderate (top right figure), and slowest (bottom figure). The figure ignores tests with run time smaller than one-tenth fraction of a second.

\section{CONCLUSION}

We presented results of validation of a new successive orders of scattering monochromatic RT code, SORD. To test the code, we used 44 benchmarks, 39 of which come from peer reviewed journals. The benchmarks provide accurate results for intensity and polarization at 16888 points. These points correspond to different geometries of observation and irradiation of a plane-parallel atmosphere-surface system and for variety of atmospheric and surface properties. Based on accuracy of modern photopolarimeters, we set $0.1 \%$ error threshold for both intensity and degree of linear polarization. SORD met the accuracy requirement in $97 \%$ cases for intensity and in $99 \%$ cases for degree of linear polarization. We noticed that convergence of intensity guarantees convergence of degree of linear polarization at least to the same level of relative accuracy.

We also reported run time for all the tests on two different machines. As expected, performance of the method of successive orders noticeably depends on the scalar (dot) product function. For best performance, the user should select the best implementation of dot product on his particular machine. In the paper, we provided some recommendations regarding intrinsic and external dot product functions in Fortran.

Based on our analysis, we conclude with confidence that SORD is a reliable RT code, which one can use for pure research (forward problems) and application (inverse problems). We created SORD in Fortran 90/95 with efficiency in mind. The code is publicly available from ftp://climate1.gsfc.nasa.gov/skorkin/SORD/ (current release is SORD_IP_16A) or by email request from corresponding (first) author. 


\section{ACKNOWLEDGEMENTS}

This research is supported by the NASA ROSES-14 program "Remote Sensing Theory for Earth Science" managed by Dr. Lucia Tsaoussi, grant number NNX15AQ23G. We are thankful to Drs. Pavel Litvinov and Oleg Dubovik for making their modification of the successive orders of scattering RT code ${ }^{13}$ available to us.

\section{REFERENCES}

[1] van de Hulst, H. C., [Multiple light scattering. Tables, formulas, and applications], Academic Press, New York (1980). Volume 1, Section 4.3, p.46;

[2] Lenoble, J. (Ed.), [Radiative Transfer in Scattering and Absorbing Atmospheres: Standard Computational Procedures], A. Deepak Publishing, Hampton VA (1985), Section 3.7, p.46;

[3] Holben, B. N., Eck, T. F., Slutsker, I., Tanré, D., Buis, J. P., Setzer, A., Vermote, E., Reagan, J. A., Kaufman, Y. J., Nakajima, T., Lavenu, F., Jankowiak, I., and Smirnov, A., "AERONET-A Federated instrument Network and Data Archive for Aerosol Characterization”, Rem. Sens. Env. 66, 1-16 (1998).

[4] Hansen, J.E., and Travis, L.D., "Light scattering in planetary atmospheres", Space Sci. Rev. 16, 265-268 (1974).

[5] Mishchenko, M. I., Lacis, A. A. and Travis, L. D., "Errors induced by the neglect of polarization in radiance calculations for Rayleigh-scattering atmospheres", J. Quant. Spect. Rad. Trans. 51(3), 491-510 (1994).

[6] Lyapustin, A., Wang, Y., Xiong, X., Meister, G., Platnik, S., Levy, R., Franz, B., Korkin, S., Hilker, T., Tucker, J., Hall, F., Sellers, P., Wu, A., and Angal, A., "Scientific impact of MODIS C5 calibration degradation and C6+ improvements", Atm. Meas. Tech. 7, 4353-4365 (2014). Fig. 6, p. 4358.

[7] Hasekamp, O. P., and Landgraf, J., "Retrieval of aerosol properties over land surfaces: capabilities of multipleviewing-angle intensity and polarization measurements", Appl. Opt. 46(16), 3332-3344 (2007).

[8] Mishchenko, M. I., Cairns, B., Kopp, G., Schueler, C. F., Fafaul, B. A., Hansen, J. E., Hooker. R. J., Itchkawich T., Maring, H. B., and Travis, L. D., "Accurate monitoring of terrestrial aerosols and total solar irradiance", Bull. Amer. Met. Soc. 88(5), 677-691 (2007).

[9] Diner, D. J., Davis, A., Hancock, B., Gutt, G., Chipman, R. A., and Cairns, B., "Dual-photoelastic-modulator-based polarimetric imaging concept for aerosol remote sensing", Appl. Opt. 46(35), 8428-8445 (2007).

[10] Chandrasekhar, S., [Radiative transfer], Oxford University Press, London (1950).

[11]Zhai, P.-W., Hu, Y., Josset, D. B., Trepte, C. R., and Lucker, P. L., "Advanced angular interpolation in the vector radiative transfer for coupled atmosphere and ocean system", J. Quant. Spect. Rad. Trans. 115, 19-27 (2013).

[12] Min, Q. and Duan, M., "A successive order of scattering model for solving vector radiative transfer in the atmosphere", J. Quant. Spect. Rad. Trans. 87, 243-259 (2004).

[13] Lenoble, J., Herman, M., Deuzé, J L., Lafrance, B., Santer, R., and Tanré, D., “A successive order of scattering code for solving the vector equation of transfer in the earth's atmosphere with aerosols", J. Quant. Spect. Rad. Trans. 107, 479-507 (2007).

[14]Duan, M. and Min, Q., "A semi-analytic technique to speed up successive order of scattering model for optically thick media”, J. Quant. Spect. Rad. Trans. 95, 21-32 (2005).

[15]Zhai, P.-W., Hu, Y., Trepte, C. R., and Lucker, P. L., "A vector radiative transfer model for coupled atmosphere and ocean systems based on successive order of scattering", Opt. Exp. 17(4), 2057-2079 (2009).

[16]Zhai, P.-W., Hu, Y., Chowdhary, J. C., Trepte, C. R., Lucker, P. L., and Josset D. B., "A vector radiative transfer model for coupled atmosphere and ocean systems with a rough surface", J. Quant. Spect. Rad. Trans. 111, 10251040 (2010).

[17]Zhai, P.-W., Hu, Y., Trepte, C. R., Lucker, P. L., and Josset, D. B., "Decoupling error for the atmospheric correction in ocean color remote sensing", J. Quant. Spect. Rad. Trans. 111, 1958-1963 (2010).

[18]Zhai, P.-W., Hu, Y., Josset, D. B., Trepte, C. R., Lucker, P. L., and Lin, B., "Exact first order scattering correction for vector radiative transfer in coupled atmosphere and ocean systems", Proc. SPIE 8364, 83640A (2012).

[19] Emde, C., Barlakas, V., Cornet, C., Evans, F., Korkin, S., Ota, Y., C.-Labonnote, L., Lyapustin, A., Macke, A., Mayer, B., and Wendisch, M., "IPRT polarized radiative transfer model intercomparison project - Phase A", J. Quant. Spect. Rad. Trans. 164, 8-36 (2015).

[20] ftp://climate1.gsfc.nasa.gov/skorkin/IPOL/ or request by email: sergey.v.korkin@ nasa.gov (9 March 2016). 
[21] Kokhanovsky, A.A., Budak, V.P., Cornet, C., Duan, M., Emde, C., Katsev, I.L., Klyukov, D.A., Korkin, S.V., C.Labonnote, L., Mayer, B., Min, Q., Nakajima, T., Ota, Y., Prikhach, A., Rozanov, V., Yokota, T. and Zege, E.P., "Benchmark results in vector atmospheric radiative transfer", J. Quant. Spect. Rad. Trans. 111, 1931-1946 (2010).

[22] http://www.meteo.physik.uni-muenchen.de/ iprt/doku.php (9 March 2016)

[23] Cox, C., and Munk, W., "Measurements of the roughness of the sea surface from photographs of the Sun's glitter", J. Opt. Soc. America 44, 838-850 (1954).

[24] Nakajima, T., and Tanaka, M., "Effect of wind-generated waves on the transfer of solar radiation in the atmosphereocean system", J. Quant. Spect. Rad. Trans. 29, 521-537 (1983).

[25] Lyapustin, A.I., "Radiative transfer code SHARM for atmospheric and terrestrial applications", Appl. Opt. 44(36), 7764-7772 (2005). See p.7770, Appendix B.

[26] Rahman, H., Pinty, B., and Verstraete, M. M., "Coupled surface-atmosphere reflectance (CSAR) model. 2. Semiempirical surface model usable with NOAA advanced very high resolution radiometer data", J. Geophys. Res. 98(20), 791-801 (1993).

[27] Nadal, F., and Bréon, F.-M., "Parameterization of surface polarized reflectance derived from POLDER spaceborne measurements", IEEE Trans. Geos. Rem Sens. 37(3), 1709-1718 (1999).

[28] Hovenier, J.W., van der Mee C., and Domke H., [Transfer of polarized light in planetary atmospheres. Basic concepts and practical methods], Kluwer Academic Publishers, Dordrecht, Netherlands (2004).

[29] Nakajima, T., and Tanaka, M., "Algorithms for radiative intensity calculations in moderately thick atmospheres using a truncation approximation”, J. Quant. Spect. Rad. Trans. 40(1), 51-69 (1988).

[30] Korkin, S. V., Lyapustin, A. I., and Rozanov, V. V., "Modifications of discrete ordinate method for computations with high scattering anisotropy: comparative analysis", J. Quant. Spect. Rad. Trans. 113, 2040-2048 (2012).

[31] Rozanov, V. V., and Lyapustin, A. I., "Similarity of radiative transfer equation: error analysis of phase function truncation techniques", J. Quant. Spect. Rad. Trans. 111, 1964-1979 (2010).

[32] Evans, K.F. and Stephens, G.L., "A new polarized atmospheric radiative transfer model”, J. Quant. Spect. Rad. Trans. 46(5), 413-423 (1991).

[33] Mishchenko, M. I., "The fast invariant imbedding method for polarized light: computational aspects and numerical results for Rayleigh scattering”, J. Quant. Spect. Rad. Trans. 43(2), 163-171 (1990).

[34] Korkin, S. V., Lyapustin, A. I. and Rozanov V. V., "APC: A new code for atmospheric polarization computations", J. Quant. Spect. Rad. Trans. 127, 1-11 (2013).

[35] de Haan, J. F., Bosma, P. B., and Hovenier, J. W., "The adding method for multiple scattering calculations of polarized light", Astron. Astroph. 183, 371-391 (1987).

[36] Wauben, W. M. F., de Haan, J. F. and Hovenier, J. W., "Low orders of scattering in plane-parallel homogeneous atmosphere", Astron. Astrophys.. 276, 589-602 (1993).

[37] Garcia, R. D. M, and Siewert, C. E., "The F_N method for radiative transfer models that include polarization effects", J. Quant. Spect. Rad. Trans. 41(2), 117-145 (1989).

[38] Wauben W. M. F, and Hovenier J. W., "Polarized radiation of an atmosphere containing randomly-oriented spheroids", J. Quant. Spect. Rad. Trans. 47(6), 491-504 (1992).

[39] Siewert, C. E., "A discrete-ordinates solution for radiative-transfer models that include polarization effects, J. Quant. Spect. Rad. Trans. 64, 227-254 (2000).

[40] Garcia, R. D. M., and Siewert, C. E., "A simplified implementation of the discrete-ordinates method for a class of problems in radiative transfer with polarization”, J. Quant. Spect. Rad. Trans. 112, 2801-2813 (2011).

[41] Wauben, W. M. F., de Haan, J. F. and Hovenier, J. W., "A method for computing visible and infrared polarized monochromatic radiation in planetary atmospheres", Astron. Astroph. 282, 277-290 (1994).

[42] Coulson, K. L., Dave, J. V., and Sekera, Z., [Tables Related to Radiation Emerging From a Planetary Atmosphere with Rayleigh Scattering], Univ. California Press, Berkeley CA (1960).

[43] Nataraj V., Li, K.-F., and Young, Y. L., "Rayleigh scattering in planetary atmopsheres: corrected tables through accurate computation of X and Y functions", Astrophys. J. 691, 1909-1920 (2009).

[44] http://www.netlib.org/lapack/explore-html/d5/df6/ddot_8f_source.html (9 March 2016)

[45] Dowd, K., [High performance computing], O’Reilly \& Associates, Inc., Sebastopol CA (1993). 